\title{
MIXED SYMMETRY-TIPE $(k, 1)$ MASSLESS TENSOR FIELDS. CONSISTENT INTERACTIONS OF DUAL LINEARIZED GRAVITY
}

\author{
C. Bizdadea, S. O. Saliu, M. Toma \\ Department of Physics, University of Craiova, 200585 Craiova, Romania
}

\section{Article Info}

Received: 29 December 2011 Accepted: 31 January 2012

Keywords: BRST symmetry, consistent interactions, dual formulations of linearized gravity.

\begin{abstract}
A particular case of interactions of a single massless tensor field with the mixed symmetry corresponding to a two-column Young diagram $(\mathrm{k}, 1)$ with $\mathrm{k}=4$, dual to linearized gravity in $D=7$, is considered in the context of: self-couplings, cross-interactions with a Pauli-Fierz field, crosscouplings to purely matter theories, and interactions with an Abelian 1form. The general approach relies on the deformation of the solution to the master equation from the antifield-BRST formalism by means of the local cohomology of the BRST differential.
\end{abstract}

\section{Introduction}

Tensor fields in "exotic" representations of the Lorentz group, characterized by a mixed Young symmetry type [1]-[5] are important given their presence in superstring, super-gravity, and super-symmetric high spin theories. They exhibit several interesting properties, such as being dual to spin two or higher field theories or not allowing consistent self-interactions in one of the dual formulation of linearized gravity (LG) [6]. An important issue related to mixed symmetry-type tensor fields is the construction of their consistent interactions in the framework of the deformation of the solution to the master equation [7] based on the computation of the local BRST cohomology [8,9]. Some recent results on this aspect are given in [10]-[14].

This work proposes the investigation of the couplings between one of the dual formulations of LG, namely that based on a massless tensor field $t_{\lambda \nu \rho \sigma \mid \alpha}$ that transforms in an irreducible representation of $G L(D, \square$ corresponding to a two-column Young diagram with 5 cells and 2 rows, and other field theories: self-couplings, cross-interactions with a PauliFierz field, cross-couplings to purely matter theories, and interactions with an Abelian vector 
field. The emerging findings emphasize no-go results in the first three situations and yes-go results for the last two cases. They generalize our previous results from [11] and [12] on the interactions involving a single massless tensor field with the mixed symmetry $(3,1)$.

\section{No-go results on couplings to itself, a spin-two field and an arbitrary matter theory}

We consider a free, massless tensor field $t_{\lambda \mu \nu \rho \mid \alpha}$ that transforms in an irreducible representation of $G L(D, \square$, corresponding to a two-column Young diagram with 5 cells and 2 rows, with the Lagrangian action

$$
\begin{aligned}
& S_{0}^{t}\left[t_{\lambda v \rho \sigma \mid \alpha}\right]=-\frac{1}{2 \cdot 4 !} \int d^{D} x\left[\left(\partial_{\mu} t_{\lambda v \rho \sigma \mid \alpha}\right) \partial^{\mu} t^{\lambda v \rho \sigma \mid \alpha}-\left(\partial^{\alpha} t_{\lambda v \rho \sigma \mid \alpha}\right) \partial_{\beta} t^{\lambda v \rho \sigma \mid \beta}\right. \\
& \left.-4\left(\partial^{\lambda} t_{\lambda \mu v \sigma \mid \alpha}\right) \partial_{\rho} t^{\rho \mu v \sigma \mid \alpha}-k\left(\partial_{\mu} t_{v \rho \sigma}\right) \partial^{\mu} t^{v \rho \sigma}-8\left(\partial^{\alpha} t_{\mu v \rho \sigma \mid \alpha}\right) \partial^{\mu} t^{\nu \rho \sigma}+12\left(\partial^{\lambda} t_{\lambda \mu \nu}\right) \partial_{\rho} t^{\rho \mu \nu}\right]
\end{aligned}
$$

This tensor field is endowed the mixed symmetry $(4,1)$, i.e. $t_{\lambda v \rho \sigma \mid \alpha}$ is antisymmetric in its first 4 indices and satisfies the (algebraic) Bianchi I identity $t_{[\lambda v \rho \sigma \mid \alpha]} \equiv 0$. The symbol $[\mu \ldots$. signifies the operation of complete antisymmetry with respect to the indices between brackets, defined such as to include only once the distinct terms. We assume a Minkowskiflat space-time of dimension $D$, endowed with a metric tensor of 'mostly plus' signature, $\sigma_{\mu v}=\sigma^{\mu v}=\operatorname{diag}\left(-+\ldots\right.$, . The trace of this tensor field is defined by $t_{\lambda v \rho}=t_{\lambda v \rho \sigma \mid \alpha} \sigma^{\sigma \alpha}$ and it is a completely antisymmetric tensor field. A generating set of gauge transformations for action (1) reads as

$$
\delta_{\theta, \varepsilon} t_{\lambda v \rho \sigma \mid \alpha}=\partial_{[\lambda} \theta_{v \rho \sigma] \alpha}+\partial_{[\lambda} \varepsilon_{v \rho \sigma] \alpha}-4 \partial_{\alpha} \varepsilon_{\lambda v \rho \sigma},
$$

where the gauge parameters are bosonic, with $\varepsilon_{\lambda v \rho \sigma}$ antisymmetric and $\theta_{v \rho \sigma \mid \alpha}$ with the mixed symmetry $(3,1)$. The space-time dimension satisfies $D \geq 6$ such that theory (1) possesses a non-negative number of physical degrees of freedom. The gauge symmetries (2) are Abelian and off-shell reducible of order 3 . This free theory, describing a massless tensor field with the mixed symmetry $(4,1)$, is dual to $L G$ in $\mathrm{D}=7$ space-time dimensions.

Related to the self-interactions of this theory, we compute the local BRST cohomology of this model and apply the procedure based on the deformation of the solution to the classical master equation. Finally, we find a no-go result, given in the next theorem.

Theorem 1 Under the assumptions of analyticity in the coupling constant, locality, Lorentz covariance, Poincaré invariance and at most two derivatives in the Lagrangian density, there are no consistent self-couplings that can be added to the free action (1) of a massless tensor field with the mixed symmetry $(4,1)$. 
The next problem is given by the consistent couplings between this tensor field and the Pauli-Fierz model (the free limit of the Hilbert-Einstein action). In this case the free Lagrangian action is written as the sum between action (1) and that of the Pauli-Fierz model and is subject to a generating set of gauge transformations listed below

$$
\begin{aligned}
& S_{0}\left[t_{\lambda \mu \nu \rho \mid \alpha}, h_{\mu v}\right]=S_{0}^{t}\left[t_{\lambda \mu \nu \rho \mid \alpha}\right]+S_{0}^{P F}\left[h_{\mu v}\right], \\
& S_{0}^{P F}\left[h_{\mu v}\right]=\int d^{D} x\left[-\frac{1}{2}\left(\partial_{\rho} h_{\mu v}\right) \partial^{\rho} h^{\mu v}+\left(\partial^{\rho} h_{\rho \mu}\right) \partial_{\lambda} h^{\lambda \mu}-\left(\partial_{\mu} h\right) \partial_{\lambda} h^{\lambda \mu}+\frac{1}{2}\left(\partial_{\mu} h\right) \partial^{\mu} h\right], \\
& \delta_{\theta, \varepsilon} t_{\lambda v \rho \sigma \mid \alpha}=\partial_{[\lambda} \theta_{v \rho \sigma] \alpha}+\partial_{[\lambda} \varepsilon_{v \rho \sigma] \alpha}-4 \partial_{\alpha} \varepsilon_{\lambda v \rho \sigma}, \quad \delta_{\xi} h_{\mu \nu}=\partial_{(\mu} \xi_{v)} .
\end{aligned}
$$

We denoted by $h$ the trace of $h_{\mu \nu}$. In the above $\xi_{\mu}$ stand for some bosonic vector gauge parameters. Acting according to the deformation procedure based on local BRST cohomology, we find the next no-go result.

Theorem 2 Under the assumptions of analyticity in the coupling constant locality, Lorentz covariance, Poincaré invariance and at most two derivatives in the Lagrangian, there are no consistent cross-interactions between a massless tensor field with the mixed symmetry $(4,1)$ and a graviton.

The only possible interacting terms that can be added to action (3) lead to the EinsteinHilbert action, invariant under diffeomorphisms.

The next task is to infer all consistent interactions between the tensor field $t_{\lambda v \rho \sigma \mid \alpha}$ and a generic matter theory. Consequently, we start from the Lagrangian action

$$
S_{0}\left[t_{\lambda v \rho \sigma \mid \alpha}, y^{i}\right]=S_{0}^{t}\left[t_{\lambda v \rho \sigma \mid \alpha}\right]+S_{0}^{\text {matt }}\left[y^{i}\right], \quad S_{0}^{\text {matt }}\left[y^{i}\right]=\int d^{D} x L\left(\left[y^{i}\right]\right),
$$

where $S_{0}^{\text {matt }}\left[y^{i}\right]$ denotes the action of a generic matter theory, whose Lagrangian density can involve at most the second-order space-time derivatives of the matter field. It is understood that all $y^{i}$ are assumed to display only purely trivial gauge symmetries, such that the gauge transformations of action (5) are given in (2) and $\delta_{\theta, \varepsilon} y^{i}=0$.

Based on cohomological arguments, it is possible to show that a necessary condition for the existence of cross-couplings at order one in the coupling constant is that the matter action displays a non-trivial rigid symmetry, with rigid parameters expressed by the components of a bosonic, completely antisymmetric and constant tensor of order $5, \xi^{\lambda \mu v \kappa \sigma}$. Let us denote by $j_{\lambda \mu \nu \kappa \sigma}^{\rho}$ the corresponding (non-trivial) conserved current, following from Noether's theorem. Consequently, we can write that 


$$
\begin{aligned}
& \Delta y^{i}=g_{\lambda \mu \nu \kappa \sigma}^{i}\left(y^{j}, \partial y^{j}\right) \xi^{\lambda \mu v \kappa \sigma}, \quad \varepsilon\left(g_{\lambda \mu \nu \kappa \sigma}^{i}\right)=\varepsilon\left(y^{i}\right) \equiv \varepsilon_{i}, \quad \varepsilon\left(\xi^{\lambda \mu \nu \kappa \sigma}\right)=0, \\
& \frac{\delta^{R} L}{\delta y^{i}} g_{\lambda \mu \nu \kappa \sigma}^{i}\left(y^{j}, \partial y^{j}\right)=\partial_{\rho} j_{\lambda \mu v \kappa \sigma}^{\rho}\left(y^{j}, \partial y^{j}\right), \quad \varepsilon\left(j_{\lambda \mu v \kappa \sigma}^{\rho}\right)=0 .
\end{aligned}
$$

Here and in the sequel $\varepsilon(F)$ denotes the Grassmann parity of $F$. Presuming this is indeed the case, it follows that both the deformed Lagrangian density and gauge transformations at order one in the coupling constant are expressed by

$$
L_{1}=\frac{1}{4} j_{\lambda \mu v \kappa \sigma}^{\rho}\left(y^{j}, \partial y^{j}\right) \partial_{\rho}^{[\lambda} t_{\rho v \kappa \sigma]}, \quad \bar{\delta}_{1 \varepsilon, \theta} y^{i}=g_{\lambda \mu \nu \kappa \sigma}^{i}\left(y^{j}, \partial y^{j}\right) \partial^{[\lambda} \varepsilon^{\mu \nu \kappa \sigma]} .
$$

The consistency of (7) at order two in the deformation parameter imposes further restrictions on $g_{\lambda \mu \nu \kappa \sigma}^{i}$, whose solutions are

$$
D=7, \quad g_{\lambda \mu \nu \kappa \sigma}^{i}\left(y^{j}, \partial y^{j}\right)=c \varepsilon_{\lambda \mu v \kappa \sigma \alpha \beta} x^{[\alpha} \partial^{\beta]} y^{i} .
$$

Due to (8) the accompanying Lagrangian density breaks the hypothesis on the Poincaré invariance of interactions, such that these couplings must be ruled out. We can consequently synthesize the previous results in the following theorem.

Theorem 3 Under the assumptions of analyticity in the coupling constant, locality, Lorentz covariance, Poincaré invariance and the preservation of the maximum derivative order of both the Lagrangian and field equations, there are no consistent cross-couplings of a massless tensor field with the mixed symmetry $(4,1)$ to a generic matter theory.

\section{Yes-go results on cross-couplings to Abelian vector fields}

The starting point of this section is given by a free model describing a massless tensor field with the mixed symmetry $(4,1)$ and an Abelian vector field

$$
\begin{aligned}
& S_{0}\left[t_{\lambda \mu \nu \rho \mid \alpha}, A_{\mu}\right]=S_{0}^{t}\left[t_{\lambda \mu v \rho \mid \alpha}\right]-\frac{1}{4} \int d^{D} x\left(F_{\mu v} F^{\mu v}\right), \\
& F_{\mu v}=\partial_{[\mu} A_{\mu]}, \quad D \geq 6, \quad O_{r e d}=3, \\
& \delta_{\theta, \varepsilon} t_{\lambda \mu v \rho \mid \alpha}=\partial_{[\lambda} \theta_{\mu v \rho] \alpha}+\partial_{[\lambda} \varepsilon_{\mu v \rho] \alpha}-4 \partial_{\alpha} \varepsilon_{\lambda \mu v \rho}, \quad \delta_{\xi} A_{\mu}=\partial_{\mu} \xi .
\end{aligned}
$$

The gauge parameter $\xi$ involved in the gauge transformation of the vector field is bosonic and defines the usual $U(1)$ gauge invariance of the Maxwell action. The field strength of the vector potential $A_{\mu}$ is denoted by $F_{\mu \nu}$ and is defined as usually like in the second line of the above formula. The above generating set of gauge transformations is off-shell reducible of order 3 strictly due to the presence of the tensor field $t_{\lambda \mu v \mid \rho}$. 
Performing the necessary cohomological computations we are able to find in this situation a fully consistent deformed solution to the master equation that leads to crosscouplings between the two types of tensor fields.

Theorem 4 Under the assumptions of analyticity in the coupling constant, locality, Lorentz covariance, Poincaré invariance and at most two derivatives in the Lagrangian density, there appear consistent cross-couplings between a massless tensor field with the mixed symmetry $(4,1)$ and an Abelian vector field. These couplings break the PT invariance and hold only in $D=6$.

The emerging Lagrangian action contains only mixing-component terms of order one and respectively two in the coupling constant that can be written in a compact form as

$$
\bar{S}_{0}\left[t_{\lambda \mu \nu \rho \mid \alpha}, A_{\mu}\right]=S_{0}^{t}\left[t_{\lambda \mu \nu \rho \mid \alpha}\right]-\frac{1}{4} \int d^{6} x\left(\bar{F}_{\mu \nu} \bar{F}^{\mu v}\right)
$$

in terms of the deformed field strength of the vector field

$$
\bar{F}_{\mu \nu}=F_{\mu \nu}+\frac{5 \lambda}{4} \varepsilon_{\mu \nu \lambda \rho \sigma \tau} \partial^{[\theta} t_{\theta}^{\lambda \rho \sigma \tau] \mid}
$$

A generating set of deformed gauge transformations for action (10) can be taken as

$$
\bar{\delta}_{\theta, \varepsilon} t_{\lambda \mu \nu \rho \mid \alpha}=\delta_{\theta, \varepsilon} t_{\lambda \mu \nu \rho \mid \alpha}, \quad \bar{\delta}_{\theta, \varepsilon, \xi} A_{\mu}=\partial_{\mu} \xi+\lambda \varepsilon_{\mu \lambda \nu \rho \sigma \tau} \partial^{[\lambda} \varepsilon^{v \rho \sigma \tau]},
$$

and hence only the gauge symmetries of the vector field are modified during the deformation procedure. Regarding other aspects of the gauge structure of the deformed model, the gauge algebra and the reducibility relations are not modified with respect to the free theory.

\section{Conclusions}

The main conclusion of this work is that a massless tensor field with the mixed symmetry $(4,1)$, which is dual to LG in $D=7$ space-time dimensions, does not allow for consistent self-interactions, couplings to the Pauli-Fierz field and respectively matter fields, but can be consistently coupled to an Abelian vector field in $D=6$. These findings strengthen our previous results on the existence of consistent interactions of dual formulations of LG by systematically applying the deformation procedure of the solution to the classical master equation combined with specific cohomological techniques. All results are valid only under the standard hypotheses of field theory: analyticity in the coupling constant, locality, Lorentz covariance, Poincaré invariance, and the preservation of the maximum derivative order of the deformed field equations with respect to the free ones. 


\section{Acknowledgements}

One of the authors (M.T.) acknowledges partial support from the strategic grant POSDRU/88/1.5/S/49516, Project ID 49516 (2009), co-financed by the European Social Fund - Investing in People, within the Sectorial Operational Programme Human Resources Development 2007-2013.

\section{References}

[1] T. Curtright, P. G. O. Freund, Nucl. Phys. B, 172 (1980) 413;

[2] T. Curtright, Phys. Lett. B, 165 (1985) 304;

[3] C. S. Aulakh, I. G. Koh, S. Ouvry, Phys. Lett. B, 173 (1986) 284;

[4] J. M. F. Labastida, T. R. Morris, Phys. Lett. B, 180(1986) 101;

[5] J. M. F. Labastida, Nucl. Phys. B, 322 (1989) 185;

[6] X. Bekaert, N. Boulanger, M. Henneaux, Phys. Rev. D, 67 (2003) 044010;

[7] G. Barnich, M. Henneaux, Phys. Lett. B, 311 (1993) 123;

[8] G. Barnich, F. Brandt, M. Henneaux, Commun. Math. Phys., 174 (1995) 57 ;

[9] G. Barnich, F. Brandt, M. Henneaux, Commun. Math. Phys., 174 (1995) 93 ;

[10] C. Burdik, A. Pashnev, M. Tsulaia, Mod. Phys. Lett. A, 16 (2001) 731 ;

[11] C. Bizdadea, C. C. Ciobîrcă, E. M. Cioroianu, I. Negru, S. O. Saliu, S. C. Săraru, J. High Energy Phys., 10 (2003) 019 ;

[12] C. Bizdadea, D. Cornea, S. O. Saliu, J. Phys. A: Math. Theor., 41 (2008) 285202;

[13] C. Bizdadea, E. M. Cioroianu, S. O. Saliu, C. C. Ciobîrcă, I. Negru, Mixed symmetrytype tensor fields (in Romanian), Universitaria Publishing House, Craiova (2008);

[14] C. Bizdadea, M. T. Miaută, I. Negru, S. O. Saliu, L. Stanciu-Oprean, M. Toma, Phys. Ann. Univ. Craiova, 20 (part I) (2010) 127. 